\title{
Mobility of Holes in Nanometer Ge-on-Si $p$-Type Metal-Oxide-Semiconductor Field-Effect Transistors at Low Temperatures
}

\author{
I. Grigelionis ${ }^{a}$, K. Fobelets ${ }^{b}$, B. Vincent ${ }^{c}$, J. Mitard ${ }^{c}$, B. De Jaeger ${ }^{c}$, E. Simoen $^{c}$, \\ T.Y. HOFFMAN ${ }^{c}$, D. YAVORSKIY ${ }^{a}$ AND J. ŁUSAKOWSKI ${ }^{a}$ \\ ${ }^{a}$ Institute of Experimental Physics, Faculty of Physics, University of Warsaw, Hoża 69, 00-681 Warsaw, Poland \\ ${ }^{b}$ Imperial College London, Exhibition Road, SW7 2BT London, UK \\ ${ }^{c}$ IMEC, Kapeldreef 75, 3001 Leuven, Belgium
}

\begin{abstract}
We investigated magnetoresistance of $p$-type Ge-on-Si metal-oxide-semiconductor field-effect transistors in order to determine the hole mobility $\mu$ as a function of the gate polarization $\left(V_{\mathrm{G}}\right)$. Measurements were carried out at $4.2 \mathrm{~K}$ and magnetic fields up to $10 \mathrm{~T}$. The signal measured was proportional to the derivative of the transistor resistance with respect to $V_{\mathrm{G}}$. To determine the hole mobility we developed a method to treat the measured signal which is based on a numerical solution of a differential equation resulting from the theoretical description of the experimental procedure. As a result, we obtained a non-monotonic $\mu\left(V_{\mathrm{G}}\right)$ dependence which is a characteristic feature of the carrier transport in gated two-dimensional structures.
\end{abstract}

PACS: 72.20.My, 85.30.Tv

\section{Introduction}

Field-effect transistors (FETs) are basic parts of most of electronic circuits and much care is attributed to their characterisation. One of the major tasks is to determine the mobility of the carriers (electrons or holes) in the transistor channel. This becomes difficult when the length of the transistor channel falls within sub-micrometer dimensions. The main reason is that standard methods of characterization are based on measurements of the gate-channel capacitance which gets very small for short-channel FETs and is often masked by parasitic capacitances of the experimental system.

An alternative method to determine the mobility in short-channel FETs was proposed some years ago [1] and is based on measurements of the geometrical magnetoresistance of a transistor. The geometrical magnetoresistance is an increase of the resistance in the magnetic field caused by turning the direction of the current lines by the Lorentz force. This effect is best observed in short, wide samples where the Hall voltage is short-circuited by the constant potential of the source and drain electrodes. Turning the current lines in short, wide samples ("transistor geometry") is opposite to the situation in long, narrow samples ("Hall-bar geometry") where the current does not change its direction but the Hall voltage is created. More details on these classical magnetoconductivity effects can be found in [2].

The magnetoresistance method has been applied to determine the electron mobility in different types of transistors, like Si MOSFETs and GaN/AlGaN HEMTs [1, 3]. It gives an independent verification of such effects as degradation of the mobility with shortening of the channel length [4], the influence of the ballistic motion on the mobility [4] or enabled a detailed analysis of scattering mechanisms in the transistor channel [5]. This method is quick and reliable and that is why it became an important tool to investigate the carrier mobility in FETs, in particular in sub-micrometer ones.

According to theoretical considerations [2], the resistance $R$ of a transistor placed in a magnetic field $B$ perpendicular to the channel is given by

$$
R(B)=R_{0}\left(1+\mu_{\mathrm{MR}}^{2} B^{2}\right)+R_{\mathrm{A}}(B),
$$

where $R_{0}$ is the resistance of the transistor channel at $B=0, \mu_{\mathrm{MR}}$ is the magnetoresistance mobility of carriers in the channel and $R_{\mathrm{A}}(B)$ is the access resistance of the drain and source electrodes. Equation (1) states that one can estimate the mobility from the $R(B)$ dependence if the access resistance is much smaller than $R_{0}$ [1], or if it is known. However, the condition $R_{\mathrm{A}} \ll R_{0}$ is often difficult to fulfil in the case of nanometer transistors where the length of the channel is very short, and to determine $R_{\mathrm{A}}(B)$ experimentally requires measurements on test structures. For instance, to estimate $R_{\mathrm{A}}(B)$, one fabricates a series of ungated devices with the same width and different source-drain separations. Then, $R_{\mathrm{A}}(B)$ can be derived from a dependence of the resistance on the device length.

To avoid difficulties caused by the unknown value of $R_{\mathrm{A}}(B)$, one can modify the experimental procedure and 
measure a derivative of $R$ with respect to $V_{\mathrm{G}}$ which elliminates $R_{\mathrm{A}}$ [5]. The price to pay is that the measured signal is proportional to

$$
\frac{\mathrm{d} R}{\mathrm{~d} V_{\mathrm{G}}}=\frac{\mathrm{d} R_{0}}{\mathrm{~d} V_{\mathrm{G}}}\left(1+\mu_{\mathrm{AC}}^{2} B^{2}\right),
$$

where the quantity $\mu_{\mathrm{AC}}$ is called the ac magnetoresistance mobility which is related to $\mu_{\mathrm{MR}}$ via a differential equation

$$
\mu_{\mathrm{AC}}^{2}=\mu_{\mathrm{MR}}^{2}\left(1-2 \frac{\frac{1}{\mu_{\mathrm{MR}}} \frac{\mathrm{d} \mu_{\mathrm{MR}}}{\mathrm{d} V_{\mathrm{G}}}}{\frac{1}{\mu_{\mathrm{MR}}} \frac{\mathrm{d} \mu_{\mathrm{MR}}}{\mathrm{d} V_{\mathrm{G}}}+\frac{1}{p} \frac{\mathrm{d} p}{\mathrm{~d} V_{\mathrm{G}}}}\right),
$$

with $p$ denoting the carrier density in the channel. Let us note that $\mu_{\mathrm{AC}}$ is a complicated function of $\mu_{\mathrm{MR}}, p$ and their derivatives and its value can be (and usually is) quite different from that of $\mu_{\mathrm{MR}}$. For dimensional reasons, the coefficient before $B^{2}$ in Eq. (2) must be of a squared mobility, and hence its symbol $\mu_{\mathrm{AC}}$ and its name, but the form of Eq. (3) clearly shows that $\mu_{\mathrm{AC}}$ is not a mobility, but is only a function related to $\mu_{\mathrm{MR}}$.

Let us note that the denominator in the second term in Eq. (3) can change sign, thus passing through zero at a certain value of $V_{\mathrm{G}}$. This is due to the fact that the sign of the derivative $\mathrm{d} p / \mathrm{d} V_{\mathrm{G}}$ is constant in the whole range of $V_{\mathrm{G}}$ (positive or negative, depending on the type of the transistor) while $\mathrm{d} \mu_{\mathrm{MR}} / \mathrm{d} V_{\mathrm{G}}$ can change sign. This non-monotonic dependence of $\mu_{\mathrm{MR}}(p)$ (or, equivalently, $\left.\mu_{\mathrm{MR}}\left(V_{\mathrm{G}}\right)\right)$ is caused by an interplay between screening by free carriers and scattering by the interface roughness. In fact, when increasing $V_{\mathrm{G}}$, one gets a larger carrier concentration which implies a more effective screening and a higher mobility but if the concentration is too large then the quantum well, where the carriers are localized, becomes very narrow and scattering by the interface leads to mobility degradation. A zero value of the denominator in Eq. (3) leads to a singularity in measured $\mu_{\mathrm{AC}}^{2}\left(V_{\mathrm{G}}\right)$ dependence which was experimentally observed [3].

Typically, the procedure to determine $\mu_{\mathrm{MR}}$ from $\mu_{\mathrm{AC}}^{2}$ is based on fitting the experimentally determined $\mu_{\mathrm{AC}}^{2}\left(V_{\mathrm{G}}\right)$ dependence to Eq. (3). To this end, one assumes that $\mu_{\mathrm{MR}}\left(V_{\mathrm{G}}\right)$ is given by a third-order polynomial and $p\left(V_{\mathrm{G}}\right)=C\left|V_{\mathrm{G}}-V_{\mathrm{TH}}\right|$, where $V_{\mathrm{TH}}$ is the threshold voltage determined from the transfer characteristics, and $C$ is the gate-to-channel capacitance which is determined from the known transistor geometry and dielectric permeability of the barrier oxide. The third-order polynomial is chosen since it is a simple function which can describe an expected $\mu_{\mathrm{MR}}\left(V_{\mathrm{G}}\right)$ dependence in the form of a non-monotonic function asymmetrical with respect to its maximum. Applications of this procedure can be found in Ref. [3] and Ref. [5].

As one can see from Eq. (3), the function to be fitted is complicated and the fitting procedure appears to be numerically unstable. Looking for a more stable procedure to find $\mu_{\mathrm{MR}}$, we solve numerically the ordinary differential Eq. (3) which can be rewritten as

$$
\frac{\mathrm{d} \mu_{\mathrm{MR}}}{\mathrm{d} V_{\mathrm{G}}}=\mu_{\mathrm{MR}}\left(\frac{\mu_{\mathrm{MR}}^{2}-\mu_{\mathrm{AC}}^{2}}{\mu_{\mathrm{MR}}^{2}+\mu_{\mathrm{AC}}^{2}}\right) \frac{1}{p} \frac{\mathrm{d} p}{\mathrm{~d} V_{\mathrm{G}}} .
$$

Both $\mu_{\mathrm{AC}}^{2}\left(V_{\mathrm{G}}\right)$ (see Eq. (2)) and $p^{-1} \mathrm{~d} p / \mathrm{d} V_{\mathrm{G}}=\mid V_{\mathrm{G}}-$ $\left.V_{\mathrm{TH}}\right|^{-1}$ were experimentally determined and Eq. (4) was solved by the Runge-Kutta method.

\section{Experiment and results}

The transistor structure is shown in Fig. 1a. It was fabricated on a $200 \mathrm{~nm}$ thick epitaxially grown relaxed Ge on $\mathrm{Si}$ substrate. The gate stack was formed from $\mathrm{Si} / \mathrm{SiO}_{2} /$ $\mathrm{HfO}_{2} / \mathrm{TaN} / \mathrm{TiN}$. NiGe/Al metallization was done for source and drain. The length and width of the transistor gate is equal to $250 \mathrm{~nm}$ and $10 \mu \mathrm{m}$, respectively.
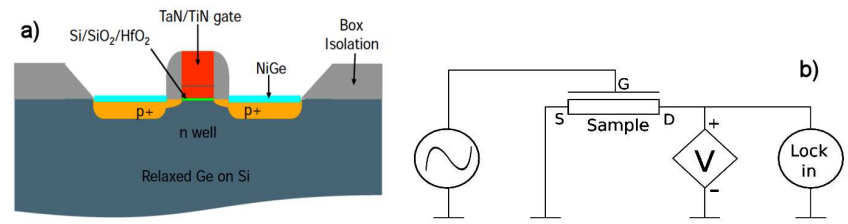

Fig. 1. (a) Schematic cross-section of the Ge on Si $p$ -MOSFET structure. (b) Electrical circuit of the measurement setup.

The transistor was placed in a liquid helium cryostat with a $10 \mathrm{~T}$ superconducting coil. Measurements were performed at $4.2 \mathrm{~K}$ using the electrical circuit shown in Fig. 1b. The transistor source was grounded, the gate was connected to a function generator, and the drain to a universal voltage source/multimeter and a lock-in. The drain was biased with a voltage $V_{\mathrm{D}}=-20 \mathrm{mV}$ and the gate with a voltage $V_{\mathrm{G}}$ from $-0.2 \mathrm{~V}$ to $-2.5 \mathrm{~V}$ with a step of $-0.1 \mathrm{~V}$. The gate potential was modulated with a sine wave with a peak-to-peak amplitude of $20 \mathrm{mV}$. In this arrangement, both the resistance $R$ (Eq. (1)) and its derivative (Eq. (2)) were measured.

Figure 2a shows experimental results for a few values of $V_{\mathrm{G}}$. In agreement with the remarks given in Introduction, one can clearly see that at $V_{\mathrm{G}}$ between $-1.5 \mathrm{~V}$ and $-1.6 \mathrm{~V}$ the measured derivative changes sign and a singularity in $\mu_{\mathrm{AC}}^{2}$ appears due to the zero value of the denominator in the second term in Eq. (3). From the fits shown in Fig. $2 \mathrm{a}, \mu_{\mathrm{AC}}^{2}$ was determined at each gate voltage and shown in Fig. 2b. The obtained dependence $\mu_{\mathrm{AC}}^{2}\left(V_{\mathrm{G}}\right)$ was approximated with a hyperbola which served as the input data for the numerical integration.

An initial condition, i.e. a value of $\mu_{\mathrm{MR}}$ at a certain $V_{\mathrm{G}}$, must be given for the integration procedure. Since no test structures were available to determine $R_{\mathrm{A}}(B)$ we assumed that for a slightly opened channel at $V_{\mathrm{G}}=-0.2 \mathrm{~V}$ (with $V_{\mathrm{TH}}=-0.15 \mathrm{~V}$ ) which contains a small concentration of holes, its resistance is much higher than the access resistance $R_{\mathrm{A}}$. In such a case, $\mu_{\mathrm{MR}}$ is given directly from dc measurements (see Eq. (1)). The final results of calculations are shown in Fig. 3. The obtained $\mu_{\mathrm{MR}}\left(V_{\mathrm{G}}\right)$ dependence shows an expected depen- 

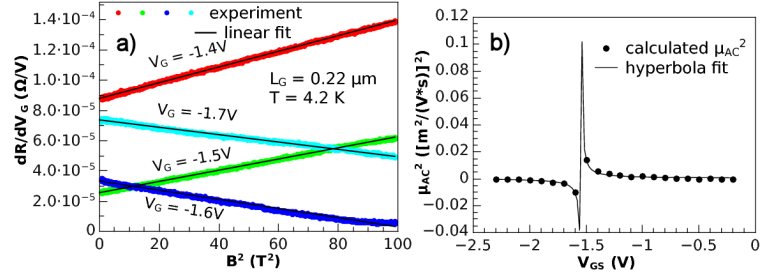

Fig. 2. (a) Examples of measured $\mathrm{d} R / \mathrm{d} V_{\mathrm{G}}(B)$ dependence at indicated $V_{\mathrm{G}}$. Symbols - experimental results, solid lines - linear fits. (b) $\mu_{\mathrm{AC}}^{2}\left(V_{\mathrm{G}}\right)$ dependence determined from data in (a). Symbols - data points obtained from (a); solid line - hyperbolic approximation.

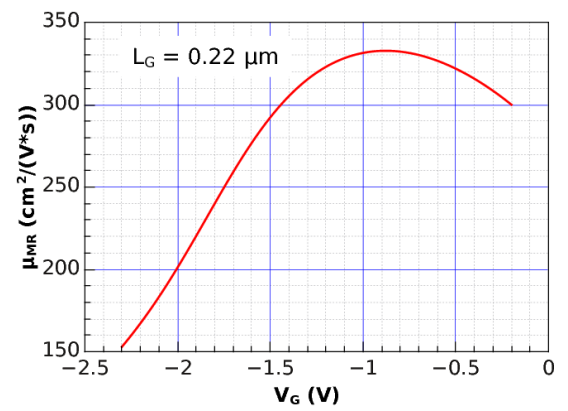

Fig. 3. Calculated $\mu_{\mathrm{MR}}\left(V_{\mathrm{G}}\right)$ dependence at $4.2 \mathrm{~K}$.

dence on $V_{\mathrm{G}}$ with the maximum mobility value of about $330 \mathrm{~cm}^{2} /(\mathrm{Vs})$ at $4.2 \mathrm{~K}$.

In conclusion, we carried out magnetotransport experiment to determine the hole mobility in a $p$-type Ge-on-
-Si MOSFETs at 4.2 K. We proposed a new method of treating the experimental data which is based on solving an appropriate differential equation. The procedure proposed is numerically stable which makes the ac magnetoresistance method even more attractive to determine the carrier mobility in short channel FETs.

\section{Acknowledgments}

This work was supported by the European Union JU ENIAC project MERCURE No. 220120.

\section{References}

[1] Y.M. Meziani, J. Łusakowski, W. Knap, N. Dyakonova, F. Teppe, K. Romanjek, M. Ferrier, R. Clerc, G. Ghibaudo, F. Boeuf, T. Skotnicki, J. Appl. Phys. 96, 5761 (2004).

[2] A C. Beer, Solid State Phys. Supplement 4 (1963).

[3] R. Tauk, J. Łusakowski, W. Knap, Z. Bougrioua, M. Azize, P. Lorenzini, M. Sakowicz, K. Karpierz, C. Fenouillet-Beranger, M. Casse, C. Gallon, F. Boeuf, T. Skotnicki, J. Appl. Phys. 102, 103701 (2007).

[4] J. Łusakowski, W. Knap, Y. Meziani, J.-P. Cesso, A. El Fatimy, R. Tauk, N. Dyakonova, G. Ghibaudo, F. Boeuf, T. Skotnicki, Appl. Phys. Lett. 87, 053507 (2005).

[5] M. Sakowicz, J. Łusakowski, K. Karpierz, M. Grynberg, B. Majkusiak, Appl. Phys. Lett. 90, 172104 (2007). 